Article

\title{
Efficient One-Pot Synthesis of Indol-3-yl-Glycines via Uncatalyzed Friedel-Crafts Reaction in Water
}

\section{Mehdi Ghandi * and Abuzar Taheri}

School of Chemistry, University College of Science, University of Tehran, Tehran, Iran

* Author to whom correspondence should be addressed; E-mail: ghandi@khayam.ut.ac.ir; Tel.: +9821-61112250; Fax: +98-21-66495291.

Received: 11 February 2009; in revised form: 25 February 2009 / Accepted: 3 March 2009 / Published: 5 March 2009

\begin{abstract}
The three component reaction of primary aliphatic amines, glyoxalic acid and indole or $\mathrm{N}$-methylindole in water at ambient temperature affords indol-3-yl or $\mathrm{N}$ methylindol-3-yl-glycine in almost quantitative yields.
\end{abstract}

Keywords: Amino acid; Indol-3-yl-glycine; Glyoxalic acid; Friedel-Crafts reaction.

\section{Introduction}

The use of water as an environmentally benign solvent for organic synthesis has become an important research area from both the economical and synthetic point of view. The indole ring system is probably the most ubiquitous heterocycle in Nature [1]. Substituted indoles have been referred to as "privileged structures" since they are able to bind with high affinity to many receptors [2]. Indol-3-ylglycine derivatives are one of the important non-proteinogenic amino acids for the synthesis of many biologically active compounds such as dragmacdins, hamacanthin and pemedolac [3-7]. Therefore, the development of new strategies to the synthesis of indol-3-yl-glycine derivatives has been the subject of considerable interest.

Owing to the importance of this class of amino acids, several procedures such as the Friedel-Crafts reaction of indole either with glyoxylate imine/iminium species or glyoxalate and amines are convenient methods for the synthesis of indol-3-yl-glycines. However, these methods in general require utilization of catalysts such as TFA, $\mathrm{Yb}(\mathrm{OTf})_{3}, 1 \mathrm{H}$-benzotriazole and $\mathrm{TiCl}_{4}$ [8-11]. Recent reports on the reaction of glyoxalic esters, amines and indole under solvent and catalyst free conditions 
are of fundamental interest [12-13]. Utilization of glyoxalic acid as aldehyde has been reported by Jiang et al., but indolyl boronic acid has been used in their approach, which does not seem to be a convenient reagent [14].

In this paper, we report the one-pot synthesis of several indol-3-yl-glycines at ambient temperature using water as solvent. The procedure is based on the uncatalyzed Friedel-Crafts condensation between indole or $\mathrm{N}$-methylindole and various iminoacids formed in situ from glyoxalic acid and primary aliphatic amines.

\section{Results and Discussion}

The model three-component reaction was carried out by stirring the mixture of indole (10 mmol), glyoxalic acid $(10 \mathrm{mmol})$ and butylamine $(10 \mathrm{mmol})$ in water $(30 \mathrm{~mL})$. It was found that at least $1 \mathrm{~h}$ is needed for the reaction to go to completion at ambient temperature. Thus, the three-component reactions of indole or $\mathrm{N}$-methylindole with glyoxalic acid and primary aliphatic amines 1a-e in water for $1 \mathrm{~h}$ afforded the indol-3-yl (2a-e) or $N$-methylindol-3-yl-glycines (3a-e), respectively. The reaction is depicted in Scheme 1 and the results are presented in Table 1.

Scheme 1. Synthesis of indol-3-yl and N-methylindol-3-yl-glycine.

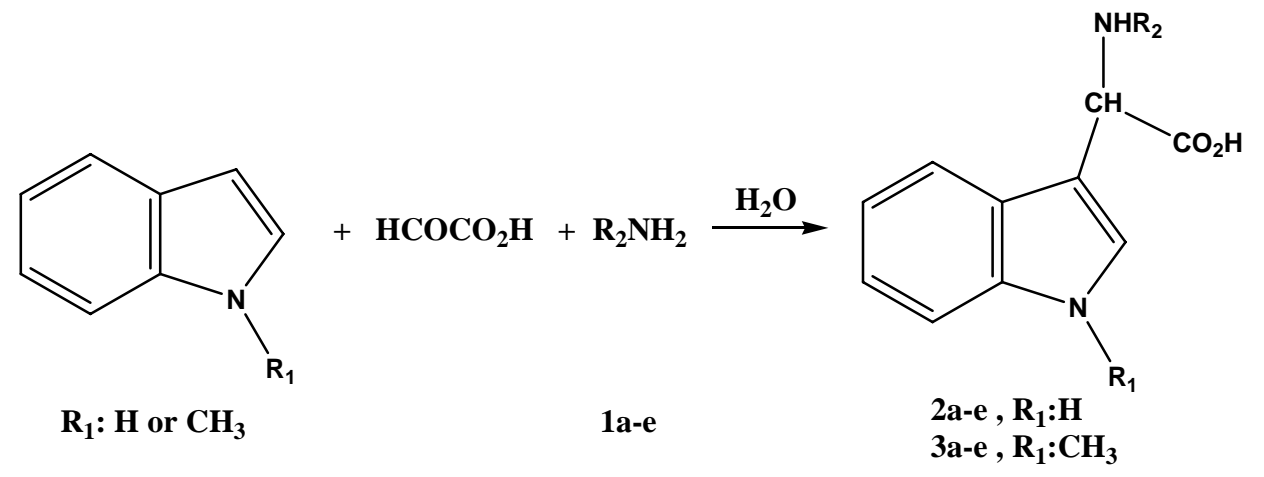

Table 1. Yield and melting points for 2a-e and 3a-e.

\begin{tabular}{ccccc}
\hline $\mathbf{R 1}$ & $\mathbf{R} 2$ & Product & $\begin{array}{c}\text { Yield } \\
\mathbf{( \% )}\end{array}$ & $\begin{array}{c}\text { M. P. } \\
\left({ }^{0} \mathrm{C}\right)\end{array}$ \\
\hline $\mathrm{H}$ & $\mathrm{CH}_{3}$ & $\mathbf{2 a}$ & 95 & $198-200$ \\
$\mathrm{H}$ & $\mathrm{CH}_{3} \mathrm{CH}_{2}$ & $\mathbf{2 b}$ & 96 & $190-191$ \\
$\mathrm{H}$ & $\mathrm{CH}_{3}\left(\mathrm{CH}_{2}\right)_{2}$ & $\mathbf{2 c}$ & 95 & $123-125$ \\
$\mathrm{H}$ & $\mathrm{CH}_{3}\left(\mathrm{CH}_{2}\right)_{3}$ & $\mathbf{2 d}$ & 94 & $214-216$ \\
$\mathrm{H}$ & $\mathrm{PhCH}_{2}$ & $\mathbf{2 e}$ & 95 & $200-201$ \\
$\mathrm{CH}_{3}$ & $\mathrm{CH}_{3}$ & 3a & 93 & $187-188$ \\
$\mathrm{CH}_{3}$ & $\mathrm{CH}_{3} \mathrm{CH}_{2}$ & 3b & 92 & $196-197$ \\
$\mathrm{CH}_{3}$ & $\mathrm{CH}_{3}\left(\mathrm{CH}_{2}\right)_{2}$ & 3c & 93 & $197-198$ \\
$\mathrm{CH}_{3}$ & $\mathrm{CH}_{3}\left(\mathrm{CH}_{2}\right)_{3}$ & 3d & 95 & $189-190$ \\
$\mathrm{CH}_{3}$ & $\mathrm{PhCH}_{2}$ & 3e & 96 & $174-176$ \\
\hline
\end{tabular}


Attempts to carry out the reaction with secondary aliphatic amines such as pyrrolidine, piperidine and diallylamine were unsuccessful, probably due to instability of the corresponding iminium salts in water. Moreover, using aromatic amines such as aniline, and 2-aminopyridine in most of the cases, messy and sticky mixtures were formed. The high efficiency of reaction might be rationalized on the basis of Brønsted acid catalysis of the carboxylic acid, as indicated in Figure 1.

Figure 1. Intramolecular acid catalysis of the Friedel-Crafts reaction of indole with an iminoacid.

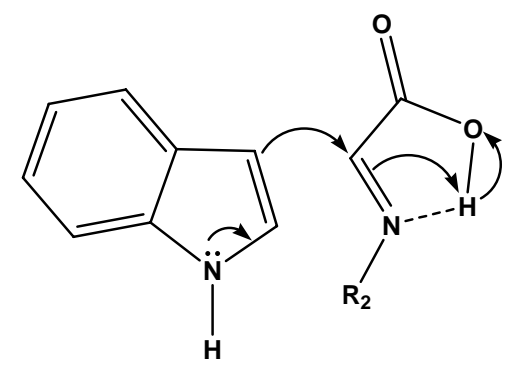

The inefficiency of aromatic amine in achieving hydrogen bonding to the acid site may be due to the weaker basic strength of the nitrogen, which is in direct conjugation with aromatic ring. Identification of the products were carried out on the basis of their spectroscopic information. For example, compound 2d exhibited a molecular ion peak at $\mathrm{m} / \mathrm{z} 246$. The IR spectrum showed the correct stretching vibrations at 2,630 $\left(\mathrm{CO}_{2} \mathrm{H}\right)$ and 1,650-1,550 $(\mathrm{C}=\mathrm{O}, \mathrm{C}=\mathrm{C}) \mathrm{cm}^{-1}$. Its ${ }^{1} \mathrm{H}-\mathrm{NMR}$ spectrum in DMSO- $\mathrm{d}_{6}$ showed a triplet at $0.80(3 \mathrm{H}, J=7.2 \mathrm{~Hz})$, a multiplet at $1.21(2 \mathrm{H})$, a multiplet at $1.56(2 \mathrm{H})$, two multiplets at 2.65 and 2.73 for diestereotopic $\mathrm{CH}_{2} \mathrm{NH}$, a singlet at $4.55(1 \mathrm{H})$, a doublet of doublets that appears as a triplet at $6.99(\mathrm{~J}=7.4 \mathrm{~Hz}, 1 \mathrm{H})$, a doublet of doublets that appears as a triplet at $7.09(\mathrm{~J}=7.5 \mathrm{~Hz}, 1 \mathrm{H})$, a doublet at $7.38(\mathrm{~J}=8.7 \mathrm{~Hz}, 1 \mathrm{H})$, a singlet at $7.39(1 \mathrm{H})$, a doublet at $7.74(\mathrm{~J}=7.8 \mathrm{~Hz}, 1 \mathrm{H})$, and a singlet at $11.33\left(1 \mathrm{H}\right.$, disappeared upon addition of $\left.\mathrm{D}_{2} \mathrm{O}\right)$. The ${ }^{13} \mathrm{C}-\mathrm{NMR}$ in $\mathrm{DMSO}_{-} \mathrm{d}_{6}$ exhibited five peaks at 14.4 to 59.25 (aliphatic carbons), eight peaks at 109.8 to 136.9 (aromatic carbons) and a peak at 169.5 (carboxylic acid $\mathrm{C}=\mathrm{O}$ ).

\section{Conclusions}

In summary, a one-pot three component reaction of indole or $N$-methylindole, glyoxalic acid and primary aliphatic amines at ambient temperature in water provides an efficient and green method for the synthesis of indol-3-yl and $N$-methylindol-3-yl-glycine.

\section{Experimental}

\section{General}

All commercially available chemicals and reagents were purchased from the Merck Company and used without further purification. Melting points were determined with an Electrothermal model 9100 apparatus and are uncorrected. IR spectra were recorded on a Shimadzu 4300 spectrophotometer. The ${ }^{1} \mathrm{H}$ - and ${ }^{13} \mathrm{C}-\mathrm{NMR}$ spectra were recorded on a Bruker DRX-500 AVANCE spectrometer. Unless otherwise specified DMSO- $\mathrm{d}_{6}$ was used as solvent. Chemical shifts $(\delta)$ were reported in ppm and 
referenced to the NMR solvent. Mass spectra of the products were obtained with a HP (Agilent technologies) 5937 Mass Selective Detector.

General procedure for the synthesis of indol-3-yl or N-methylindol-3-yl-glycines 2a-e and 3a-e

To a solution of indole or $N$-methylindole $(10 \mathrm{mmol})$ and glyoxalic acid $(10 \mathrm{mmol})$ in water $(30$ $\mathrm{mL})$ was added aliphatic amine $(10 \mathrm{mmol})$ and the mixture was stirred for $1 \mathrm{~h}$ at ambient temperature. After filtration of the precipitate formed, the solid was purified by trituration in hot methanol and then in hot ethylacetate.

Indol-3-yl-N-methylglycine (2a): White solid; IR (KBr): $3448(\mathrm{NH}), 3153,2993,2875,2528\left(\mathrm{CO}_{2} \mathrm{H}\right)$, $1645(\mathrm{C}=\mathrm{O}), 1602 \mathrm{~cm}^{-1} ;{ }^{1} \mathrm{H}-\mathrm{NMR} \delta: 2.35(\mathrm{~s}, 3 \mathrm{H}), 4.48(\mathrm{~s}, 1 \mathrm{H}), 6.99(\mathrm{t}, J=7.7 \mathrm{~Hz}, 1 \mathrm{H}), 7.08(\mathrm{t}, J=7.9$ $\mathrm{Hz}, 1 \mathrm{H}), 7.36(\mathrm{~s}, 1 \mathrm{H}), 7.37$ (d, $J=7.9 \mathrm{~Hz}, 1 \mathrm{H}), 7.74(\mathrm{~d}, J=7.7 \mathrm{~Hz}, 1 \mathrm{H}), 11.34(\mathrm{~s}, 1 \mathrm{H}) \mathrm{ppm} ;{ }^{13} \mathrm{C}-\mathrm{NMR}$ $\delta: 31.9,60.5,109.5,112.3,119.5,120.5,122.1,126.7,127.2,137.0,169.2$ ppm; MS (EI): m/z 149 $\left(\mathrm{M}^{+}-45\right)$.

Indol-3-yl-N-ethylglycine (2b): Cream solid; IR (KBr): 3514, 3186, 2877,2763, $2592\left(\mathrm{CO}_{2} \mathrm{H}\right), 1625$ $(\mathrm{C}=\mathrm{O}), 1602 \mathrm{~cm}^{-1} ;{ }^{1} \mathrm{H}-\mathrm{NMR} \delta: 1.13(\mathrm{dd}, 3 \mathrm{H}), 2.69(\mathrm{~m}, 1 \mathrm{H}), 2.80(\mathrm{~m}, 1 \mathrm{H}), 4.52(\mathrm{~s}, 1 \mathrm{H}), 6.99(\mathrm{t}, J=7.5$ $\mathrm{Hz}, 1 \mathrm{H}), 7.08(\mathrm{t}, J=7.7 \mathrm{~Hz}, 1 \mathrm{H}), 7.36-7.37$ (bd, 2H), 7.73 (d, $J=7.5 \mathrm{~Hz}, 1 \mathrm{H}), 11.24(\mathrm{~s}, 1 \mathrm{H}) \mathrm{ppm} ;{ }^{13} \mathrm{C}-$ NMR $\delta: 11.9,41.2,58.8,109.8,112.3,119.5,120.3,122.1,126.4,127.3,136.9,169.0$ ppm; MS (EI): $\mathrm{m} / \mathrm{z} 218\left(\mathrm{M}^{+}\right)$.

Indol-3-yl-N-propylglycine (2c): Cream solid; IR (KBr): 3109, 2960, 2711, $2559\left(\mathrm{CO}_{2} \mathrm{H}\right), 1645(\mathrm{C}=\mathrm{O})$, $1600 \mathrm{~cm}^{-1}$; ${ }^{1} \mathrm{H}-\mathrm{NMR} \delta: 0.79(\mathrm{t}, J=7.4 \mathrm{~Hz}, 3 \mathrm{H}), 1.59(\mathrm{~m}, 2 \mathrm{H}), 2.62(\mathrm{~m}, 1 \mathrm{H}), 2.69(\mathrm{~m}, 1 \mathrm{H}), 4.59(\mathrm{~s}, 1 \mathrm{H})$, $6.99(\mathrm{t}, J=7.5 \mathrm{~Hz}, 1 \mathrm{H}), 7.08(\mathrm{t}, J=7.8 \mathrm{~Hz}, 1 \mathrm{H}), 7.38(\mathrm{~d}, J=7.8 \mathrm{~Hz}, 1 \mathrm{H}), 7.4(\mathrm{~s}, 1 \mathrm{H}), 7.73(\mathrm{~d}, J=7.5$ $\mathrm{Hz}, 1 \mathrm{H}), 11.42(\mathrm{~s}, 1 \mathrm{H}) \mathrm{ppm} ;{ }^{13} \mathrm{C}-\mathrm{NMR} \delta: 11.9,19.8,47.9,59.1,109.5,112.4,119.5,120.2,122.0$, 126.6, 127.3, 137.0, 169.7 ppm; MS (EI): m/z $232\left(\mathrm{M}^{+}\right)$.

Indol-3-yl-N-butylglycine (2d): Light pink solid; IR (KBr): $3492(\mathrm{NH}), 3321,3060,2933,2759,2630$ $\left(\mathrm{CO}_{2} \mathrm{H}\right), 1650-1550(\mathrm{C}=\mathrm{O}, \mathrm{C}=\mathrm{C}) \mathrm{cm}^{-1} ;{ }^{1} \mathrm{H}-\mathrm{NMR} \delta: 0.80(\mathrm{t}, J=7.2 \mathrm{~Hz}, 3 \mathrm{H}), 1.21(\mathrm{~m}, 2 \mathrm{H}), 1.56(\mathrm{~m}$, 2H), $2.65(\mathrm{~m}, 1 \mathrm{H}), 2.73(\mathrm{~m}, 1 \mathrm{H}), 4.55(\mathrm{~s}, 1 \mathrm{H}), 6.99(\mathrm{t}, J=7.5 \mathrm{~Hz}, 1 \mathrm{H}), 7.09(\mathrm{t}, J=7.8 \mathrm{~Hz}, 1 \mathrm{H}), 7.38$ $(\mathrm{d}, J=7.8 \mathrm{~Hz}, 1 \mathrm{H}), 7.39(\mathrm{~s}, 1 \mathrm{H}), 7.74(\mathrm{~d}, J=7.5 \mathrm{~Hz}), 11.33(\mathrm{~s}, 1 \mathrm{H}) \mathrm{ppm} ;{ }^{13} \mathrm{C}-\mathrm{NMR} \delta: 14.4,20.3,28.4$, 46.1, 59.2, 109.9, 112.3, 119.5, 120.3, 122.0, 126.5, 127.4, 136.9, 169.5 ppm; MS (EI): m/z $246\left(\mathrm{M}^{+}\right)$.

Indol-3-yl-N-benzylglycine (2e): Dark pink solid; IR (KBr): 3373 (NH), 3213, 3109, 2991, 2493, 2629 $\left(\mathrm{CO}_{2} \mathrm{H}\right), 1650-1550(\mathrm{C}=\mathrm{O}, \mathrm{C}=\mathrm{C}) \mathrm{cm}^{-1} ;{ }^{1} \mathrm{H}-\mathrm{NMR} \delta: 3.89(\mathrm{AB}, J=13.3 \mathrm{~Hz}, 2 \mathrm{H}), 4.47(\mathrm{~s}, 1 \mathrm{H}), 6.98(\mathrm{t}, J$ $=7.3 \mathrm{~Hz}, 1 \mathrm{H}), 7.09(\mathrm{t}, J=7.6 \mathrm{~Hz}, 1 \mathrm{H}), 7.32-7.60(\mathrm{~m}, 7 \mathrm{H}), 7.60(\mathrm{~d}, J=7.6 \mathrm{~Hz}, 1 \mathrm{H}), 11.13(\mathrm{~s}, 1 \mathrm{H})$ ppm; ${ }^{13} \mathrm{C}-\mathrm{NMR} \delta: 48.8,55.3,112.4,117.7,120.5,122.8,124.9,127.7,128.9,129.5,129.7,136.0$, $170.1 \mathrm{ppm} ; \mathrm{MS}(\mathrm{EI}): \mathrm{m} / \mathrm{z} 280\left(\mathrm{M}^{+}\right)$.

N-methylindol-3-yl-N-methylglycine (3a): White solid; IR ( $\mathrm{KBr}): 3111,3003,2879,2522\left(\mathrm{CO}_{2} \mathrm{H}\right)$, 1643 (CO), $1598 \mathrm{~cm}^{-1}$; ${ }^{1} \mathrm{H}-\mathrm{NMR} \delta: 2.36(\mathrm{~s}, 3 \mathrm{H}), 3.78(\mathrm{~s}, 3 \mathrm{H}), 4.40(\mathrm{~s}, 1 \mathrm{H}), 7.04(\mathrm{t}, J=7.7 \mathrm{~Hz}, 1 \mathrm{H})$, 
$7.17(\mathrm{t}, J=7.8 \mathrm{~Hz}, 1 \mathrm{H}), 7.33(\mathrm{~s}, 1 \mathrm{H}), 7.42(\mathrm{~d}, J=7.8 \mathrm{~Hz}, 1 \mathrm{H}), 7.75(\mathrm{~d}, J=7.7 \mathrm{~Hz}, 1 \mathrm{H}) \mathrm{ppm} ;{ }^{13} \mathrm{C}-\mathrm{NMR}$ $\left(\mathrm{D}_{2} \mathrm{O}+\mathrm{HCl}\right) \delta: 30.5,32.6,57.2,100.9,110.7,118.1,120.7,122.8,125.4,132.3,137.1,170.69$ ppm; MS (EI): $\mathrm{m} / \mathrm{z} 218\left(\mathrm{M}^{+}\right)$.

$N$-methylindol-3-yl-N-ethylglycine (3b): White solid; IR (KBr): 3109, 2979, 2680, $2534\left(\mathrm{CO}_{2} \mathrm{H}\right), 1650$ (CO), $1596 \mathrm{~cm}^{-1}$; ${ }^{1} \mathrm{H}-\mathrm{NMR} \delta: 1.14(\mathrm{t}, J=7.1 \mathrm{~Hz}, 3 \mathrm{H}), 2.70(\mathrm{~m}, 1 \mathrm{H}), 2.80(\mathrm{~m}, 1 \mathrm{H}), 3.78(\mathrm{~s}, 3 \mathrm{H}), 4.46$ (s, 1H), $7.05(\mathrm{t}, J=7.5 \mathrm{~Hz}, 1 \mathrm{H}), 7.17(\mathrm{t}, J=7.8 \mathrm{~Hz}, 1 \mathrm{H}), 7.35(\mathrm{~s}, 1 \mathrm{H}), 7.42(\mathrm{~d}, J=7.8 \mathrm{~Hz}, 1 \mathrm{H}), 7.75$ $(\mathrm{d}, J=7.5 \mathrm{~Hz}, 1 \mathrm{H}) \mathrm{ppm} ;{ }^{13} \mathrm{C}-\mathrm{NMR}\left(\mathrm{D}_{2} \mathrm{O}+\mathrm{HCl}\right) \delta: 10.6,32.7,41.0,55.8,101.5,110.8,118.2,120.8$, 122.9, 125.6, 132.0, 137.1, 170.8 ppm; $\mathrm{MS}(\mathrm{EI}): \mathrm{m} / \mathrm{z} 232\left(\mathrm{M}^{+}\right)$.

N-methylindol-3-yl-N-propylglycine (3c): White solid; IR (KBr): 3111, 2966, 2825, $2549\left(\mathrm{CO}_{2} \mathrm{H}\right)$, $1630(\mathrm{CO}), 1573 \mathrm{~cm}^{-1} ;{ }^{1} \mathrm{H}-\mathrm{NMR} \delta: 0.43$ (t, $\left.J=8 \mathrm{~Hz}, 3 \mathrm{H}\right), 1.23(\mathrm{~m}, 1 \mathrm{H}), 1.3(\mathrm{~m}, 1 \mathrm{H}), 2.43(\mathrm{~m}, 1 \mathrm{H})$, $2.51(\mathrm{~m}, 1 \mathrm{H}), 3.25(\mathrm{~s}, 1 \mathrm{H}), 6.8(\mathrm{~m}, 2 \mathrm{H}), 6.91(\mathrm{~d}, J=7.8 \mathrm{~Hz}, 1 \mathrm{H}), 7.12(\mathrm{~s}, 1 \mathrm{H}), 7.28(\mathrm{~d}, J=7.6 \mathrm{~Hz}$, $1 \mathrm{H}) \mathrm{ppm} ;{ }^{13} \mathrm{C}-\mathrm{NMR}\left(\mathrm{D}_{2} \mathrm{O}+\mathrm{HCl}\right) \delta: 10.5,19.2,32.7,47.2,56.0,101.6,110.8,118.3,120.8,122.9$, 125.9, 131.9, 137.1, 170.9 ppm; MS (EI): m/z $201\left(\mathrm{M}^{+}-45\right)$.

$N$-methylindol-3-yl-N-buthylglycine (3d): Light pink solid; IR (KBr): 3111, 2934, 26940, 2549( $\left.\mathrm{CO}_{2} \mathrm{H}\right)$, $1620(\mathrm{CO}), 1596 \mathrm{~cm}^{-1}$; ${ }^{1} \mathrm{H}-\mathrm{NMR} \delta: 0.83(\mathrm{t}, J=6.5 \mathrm{~Hz}, 3 \mathrm{H}), 1.23(\mathrm{~m}, 2 \mathrm{H}), 1.55(\mathrm{~m}, 2 \mathrm{H}), 2.80(\mathrm{~m}, 2 \mathrm{H})$, $3.73(\mathrm{~s}, 3 \mathrm{H}), 4.45(\mathrm{~s}, 1 \mathrm{H}), 7.02(\mathrm{~m}, 1 \mathrm{H}), 7.14(\mathrm{~m}, 1 \mathrm{H}), 7.30(\mathrm{~s}, 1 \mathrm{H}), 7.37$ (d, J = $7.9 \mathrm{~Hz}, 1 \mathrm{H}), 7.74(\mathrm{~d}, J$ $=7.7 \mathrm{~Hz}, 1 \mathrm{H}) \mathrm{ppm} ;{ }^{13} \mathrm{C}-\mathrm{NMR}\left(\mathrm{D}_{2} \mathrm{O}+\mathrm{HCl}\right) \delta: 12.8,19.3,27.4,32.8,45.5,56.0,101.4,110.9,118.2$, 120.8, 122.9, 125.7, 132.1, 137.1, $170.8 \mathrm{ppm}$; MS (EI): m/z $260\left(\mathrm{M}^{+}\right)$.

N-methylindol-3-yl-N-benzylglycine (3e): White solid; IR (KBr): 3438, 3069, 2825, $2333\left(\mathrm{CO}_{2} \mathrm{H}\right)$, $1635(\mathrm{CO}), 1600 \mathrm{~cm}^{-1} ;{ }^{1} \mathrm{H}-\mathrm{NMR} \delta: 3.75(\mathrm{~s}, 3 \mathrm{H}), 3.88(\mathrm{AB}, J=13.42 \mathrm{H}), 4.48(\mathrm{~s}, 1 \mathrm{H}), 7.02(\mathrm{t}, J=7.5$ $\mathrm{Hz}, 1 \mathrm{H}), 7.16(\mathrm{t}, J=7.7 \mathrm{~Hz}, 1 \mathrm{H}), 7.31-7.42(\mathrm{~m}, 7 \mathrm{H}), 7.61(\mathrm{~d}, J=7.5 \mathrm{~Hz}, 1 \mathrm{H}) \mathrm{ppm} ;{ }^{13} \mathrm{C}-\mathrm{NMR} \delta: 33.3$, $50.5,58.1,110.5,119.7,120.4,122.2,127.5,128.6,129.2,129.9,136.9,137.4,171.5$ ppm; MS (EI): $\mathrm{m} / \mathrm{z} 294\left(\mathrm{M}^{+}\right)$.

\section{Acknowledgements}

The authors wish to thank the Research Council of the University of Tehran for financial support.

\section{References and Notes}

1. Humphrey, G.R.; Kuethe, T.J. Practical Methodologies for the Synthesis of Indoles. Chem. Rev. 2006, 106, 2875-2911.

2. Horton, D.A.; Bourne, G.T.; Smythe, M.L. The Combinatorial Synthesis of Bicyclic Privileged Structures or Privileged Substructures. Chem. Rev. 2003, 103, 893-930.

3. Kawasaki, T.; Enoki, H.; Matasumura, K.; Ohyama, M.; Inagawa, M.; Sakamato, M. First Total Synthesis of Dragmacidin A via Indolylglycines. Org. Lett. 2000, 2, 3027-3029.

4. Blaszczak, L.C.; Turner, J.R. Indolylglycyl cephalosporin derivatives. US Patent 4492694, 1985. 
5. Katz, A. H.; Demerson, C.A.; Shaw, C.C.; Asselin, A.A.; Humber, L.G.; Conway, K.M.; Gavin, G.; Guinosso, C.; Jensen, N.P.; Mobilio, D.; Noureldin, R.; Schmid, J.; Shah, U.V.; Engen, D.; Chau, T.T.; Weichman, B.M. Synthesis and Analgesic Activity of Pemedolac [cis- 1-Ethyl1,3,4,9-tetrahydro-4-(phenylmethyl)pyrano[3,4-b]indole-1-acetic acid]. J. Med. Chem. 1981, 31, 1244-1250.

6. Kawasaki, T.; Kouko, T.; Totsuka, H.; Hiramatsu, H. Synthesis of marine bisindole alkaloids, hamacanthins A and B through intramolecular transamidation-cyclization. Tetrahedron Lett. 2003, 44, 8849-8852.

7. Jiang, B.; Gu, X.H. Syntheses of bis(3- indolyl) 2-(1H)- pyrazinones. Heterocycles 2000, 53, 1559-1568

8. Nakata, H.; Imai, T.; Yokoshima, S.; Fukuyama, T. A Simple Chiral Template for the Synthesis of Fuctionalized $\alpha$-Arylglycine Derivatives. Heterocycles 2008, 76, 747-757.

9. Fan, R.; Li, W.; Wang, B. A one-pot oxidative decarboxylation-Friedel-Crafts reaction of acyclic $\alpha$-amino acid derivatives activated by the combination of iodobenzene diacetate/iodine and iron dust. Org. Biomol. Chem. 2008, 6, 4615-4621.

10. Grumbach, H.J.; Merla, B.; Rischo, N. Efficient synthesis of racemic $\alpha$ - aryl- $\alpha$-amino acid esters via amino alkylation with in situ generated glycine cation equivalents. Synthesis 1999, 1027-1033.

11. Clark, B.; Harris, J. Syntheses of 7-carboxyindolylglycines utilizing glycine cation equivalents. Syn. Commun. 1991, 27, 4223-4234.

12. Jiang, B.; Huang, Z.G. Synthesis of $\alpha$-(3-Indolyl) glycine Derivatives via Spontaneous FriedelCrafts Reaction between Indoles and Glyoxylate Imines. Synthesis 2005, 2198-2204.

13. Zhao, J.L.; Liu, L.; Zhang, H.B.; Wu, Y.C.; Wang, D.; Chen, Y.J. Three-Component FriedelCrafts Reaction of Indoles, Glyoxylate, and Amine under Solvent-Free and Catalyst-Free Conditions- Synthesis of (3-Indolyl) glycine Derivatives. Synlett 2006, 96-100.

14. Jiang, B.; Yang, C.G.; Gu, X.H. A highly stereoselective synthesis of indolyl $N$ - substituted glycines. Tetrahedron Lett. 2001, 42, 2545-2547.

Sample Availability: Samples of the compounds $\mathbf{2}$ and $\mathbf{3}$ are available from the authors.

(C) 2009 by the authors; licensee Molecular Diversity Preservation International, Basel, Switzerland. This article is an open-access article distributed under the terms and conditions of the Creative Commons Attribution license (http://creativecommons.org/licenses/by/3.0/). 